\title{
One Novel Hydraulic Actuating System for the Lower-Body Exoskeleton
}

\author{
Maowen Sun ${ }^{1}$, Xiaoping Ouyang ${ }^{1 *}$, Jouni Mattila², Huayong Yang ${ }^{1}$ and Gang Hou ${ }^{3}$
}

\begin{abstract}
The hydraulic exoskeleton is one research hotspot in the field of robotics, which can take heavy load due to the high power density of the hydraulic system. However, the traditional hydraulic system is normally centralized, inefficient, and bulky during application, which limits its development in the exoskeleton. For improving the robot's performance, its hydraulic actuating system should be optimized further. In this paper a novel hydraulic actuating system (HAS) based on electric-hydrostatic actuator is proposed, which is applied to hip and knee joints. Each HAS integrates an electric servo motor, a high-speed micro pump, a specific tank, and other components into a module. The specific parameters are obtained through relevant simulation according to human motion data and load requirements. The dynamic models of the HAS are built, and validated by the system identification. Experiments of trajectory tracking and human-exoskeleton interaction are carried out, which demonstrate the proposed HAS has the ability to be applied to the exoskeleton. Compared with the previous prototype, the total weight of the HAS in the robot is reduced by about $40 \%$, and the power density is increased by almost 1.6 times.
\end{abstract}

Keywords: Hydraulic actuating system (HAS), Lower-body exoskeletons, Lightweight and integrated, System identification, Working mode test

\section{Introduction}

Since 2000, exoskeletons have been widely studied, including human rehabilitation $[1,2]$ and power-assisted exoskeletons [3, 4]. The centralized hydraulic system which consists of one pump unit, multiple control valves and actuators, has been used in the exoskeletons due to its high-power density. In these robot prototypes, the centralized hydraulic system was normally bulky installed in the robots or placed outside. Such as In Raytheon's XOS prototype [5], the hydraulic power unit was installed outside of the exoskeleton, which limited the robot going freely. The BLEEX prototype $[6,7]$ hired the centralized hydraulic system powered by the gasoline engine for actuating. The HULC prototype [8] used the centralized hydraulic system, in which the hydraulic pump is driven by electric-motor to deliver fluid power

\footnotetext{
*Correspondence: ouyangxp@zju.edu.cn

1 State Key Laboratory of Fluid Power \& Mechatronic Systems, Zhejiang

University, Hangzhou 310027, China

Full list of author information is available at the end of the article
}

to the exoskeleton joints. Our group once developed a compact centralized hydraulic power unit (CHPU) for the exoskeleton $[9,10]$, which improved the power density. Centralized hydraulic systems demonstrate its high power density, but at the same time, some shortcomings hinder the development of the exoskeleton further, such as low efficiency and bulky structure. The centralized hydraulic system requires constant energy supply under working conditions, and the energy is released through the control valves, which will cause the energy loss due to the valve throttling.

Recently the electro-hydrostatic actuator (EHA), has been widely applied to advanced aircrafts [11], which demonstrated more compact structure, higher power density and efficiency compared with the centralized hydraulic systems. Many scholars have conducted research on the EHA design [12], control methods [13, 14], vibration and friction $[15,16]$. EHA have also been applied to robotics such as humanoid robot. The University of Tokyo developed an EHA actuated anthropomorphic hand [17, 18]
Springer Open

(c) The Author(s) 2021. This article is licensed under a Creative Commons Attribution 4.0 International License, which permits use, sharing, adaptation, distribution and reproduction in any medium or format, as long as you give appropriate credit to the original author(s) and the source, provide a link to the Creative Commons licence, and indicate if changes were made. The images or other third party material in this article are included in the article's Creative Commons licence, unless indicated otherwise in a credit line to the material. If material is not included in the article's Creative Commons licence and your intended use is not permitted by statutory regulation or exceeds the permitted use, you will need to obtain permission directly from the copyright holder. To view a copy of this licence, visit http://creativeco mmons.org/licenses/by/4.0/. 
and humanoid robot $[19,20]$. The Kyushu Sangyo University designed the micro EHA's actuated robotic arm [21]. And the application of EHA in wearable assistive devices has also been studied in recent years. An EHA system of enlarging the human knee joint capacity was studied in Refs. [22, 23] which realized the EHA firstly used in the assisted single joint. The pump-controlled system indeed has the potential of being the power unit in robots. But the research for pump-controlled system applied to exoskeleton has not been sufficiently studied, especially in the application of multi-joint exoskeleton under heavy load.

Considering advantage of EHA, a novel hydraulic actuating system (HAS) for the exoskeleton was proposed in this paper. Its principle and specific parameters were introduced in Section 2, and its linear dynamic model was built in Section 3. The HAS prototype, and its system identification were carried out in Section 4. Tracking performance under walking and squatting motion in the load test rig and the exoskeleton were verified respectively in Section 5.

\section{Novel HAS for the Exoskeleton}

\subsection{Overview of the HAS for the Exoskeleton}

The novel hydraulic system of the exoskeleton [24] is shown in Figure 1, which is driven by four HASs for four active joints replacing the centralized hydraulic system. Each HAS has its own motor and pump, realizing volume control, improving the energy efficiency. Compared with the XOS2 exoskeleton [5] and Berkeley lower Extremity exoskeleton [6], the HAS driven exoskeleton looks more compact.

\subsection{Working Principle}

Each HAS has similar working principle. The HAS of the hip joint is taken as an example for detailed analysis. Figure 2 shows the proposed hydraulic schematic of HAS which integrates the following components: electric servo motor (ESM) (a), bidirectional piston pump (b), flow matching valve (FMV) (e), directional control valve (DCV) (c), safety valve (d), elastic tank (f), and cylinder(g).

The rodless and rod chambers of the cylinder are connected with both DCV (c) and safety valve (d). The position of the FMV (e) spool depends on the pressure difference between the inlet and outlet port of the pump. The FMV (e) realizes the switching of the system oil supply. Extension motion of the cylinder occurs when the fluid is transmitted into the rodless chamber through the DCV (c). The fluid from the rod chamber returns to the tank through the DCV (c), and any excess fluid goes back into the tank. The motion direction of the cylinder is changed by switching the rotation direction of ESM (a). Once the ESM (a) stops, the cylinder is free and driven by the external force.
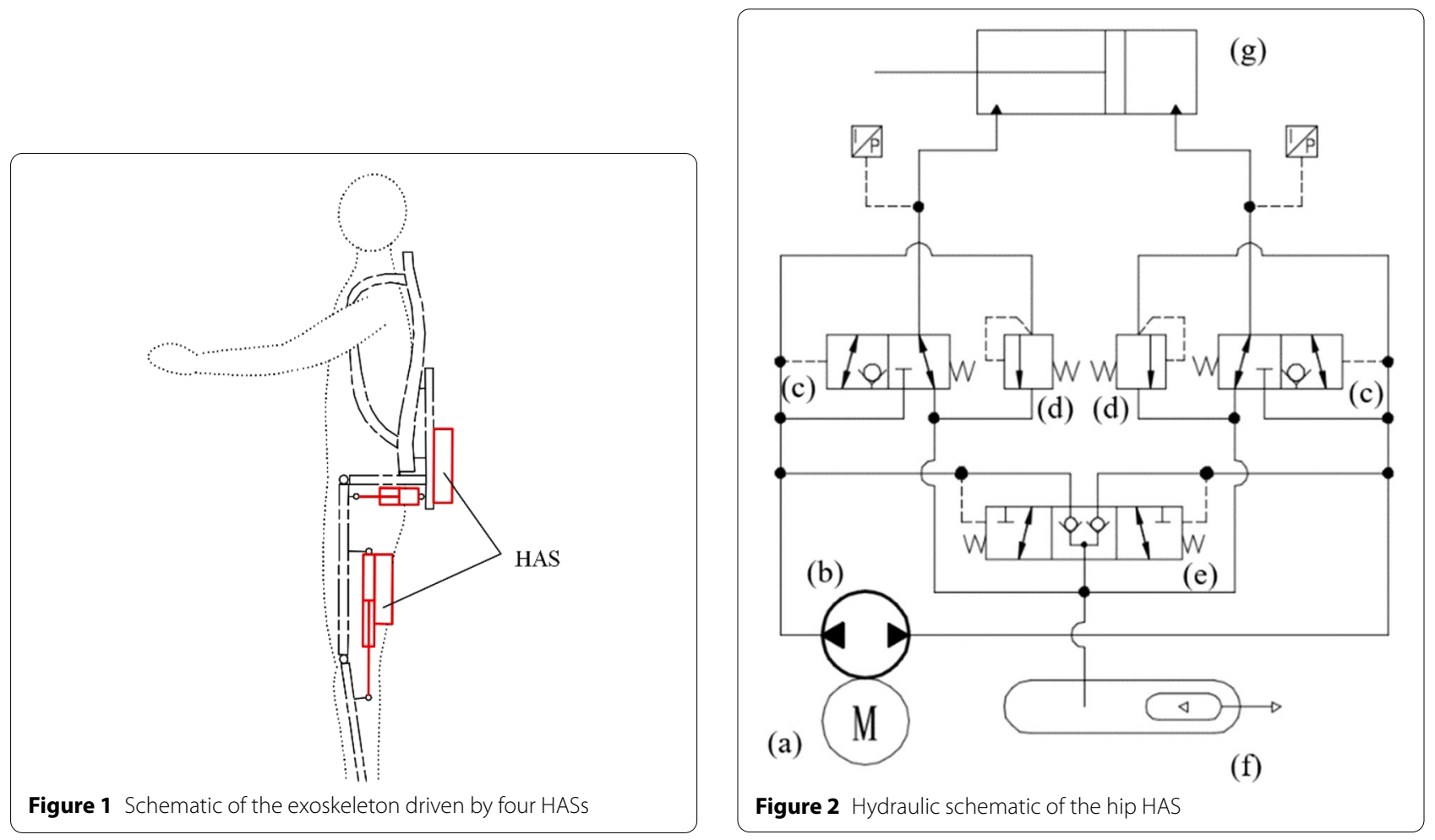


\subsection{Key Components}

\subsubsection{Micro Pump}

A high-power density, seven-piston fixed displacement bidirectional piston pump for the HAS is developed, shown in Figure 3. The special corrugated spring is used to reduce the layout space of the piston return mechanism. And the spherical contact between the cylinder block and the port plate which reduces inner leakage, the volumetric efficiency is improved [25]. Its displacement is $0.4 \mathrm{~mL} / \mathrm{r}$, the maximum working pressure can reach up to $18 \mathrm{MPa}$, and the required rotate speed up to $4000 \mathrm{r} / \mathrm{min}$. The pump weights $200 \mathrm{~g}$, and its size is only $59 \mathrm{~mm} \times 59$ $\mathrm{mm} \times 92 \mathrm{~mm}$.

\subsubsection{The Specific Tank}

In order to reduce the volume of the HAS, a non-pressurized and compact tank is developed, and its structure is shown in Figure 4. The oil is filled in the chamber between the shell and the soft rubber skin. The soft rubber skin isolates the external environment. And there are six vents on the top of the tank, so the shape of the soft rubber skin changes with the oil volume. Inlet and outlet ports are located on the bottom of the tank.

\subsubsection{Valve Block}

The valve block of the HAS includes FMV (e), DCV (c) and safety valve $(\mathrm{d})$. Figure 5 shows one of the working mode of FMV (e). The valve is symmetrical, and the two ports are respectively connected to the inlet and outlet of the pump. When the port 2 is connected to the outlet of the pump, high pressure oil will be injected into the right valve cavity (shown as red arrow). The valve spools (L1) and (L2) will be pushed by the valve spool (R2), and the right valve chamber will be closed by the valve spool (R2). The oil from the tank inlet port will enter into the pump inlet port through the port 1 (show as blue arrow). The FMV (e) solves the problem of the asymmetric flow matching, and weights only $22 \mathrm{~g}$.

To reduce the volume and weight, the DCV (c) and the safety valve (d) are integrated into the one compact valve, as shown in Figure 6. The outlet port 1 is connected to the chamber of the cylinder, the outlet port 2 is connected to the tank, and the inlet port is connected to the outlet of the pump. The compact valve has three working modes for achieving three working states of the exoskeleton.

(I) When the ESM (a) stops, there is no pressure in circuit, the outlet port 1 is connected to the outlet port 2 . At this time, the oil between the cylinder and the tank circulates freely (shown in Figure 6(a)), and the cylinder can be moved freely. (II) When the system pressure is established, the valve spool 1 and the valve spool 2 are

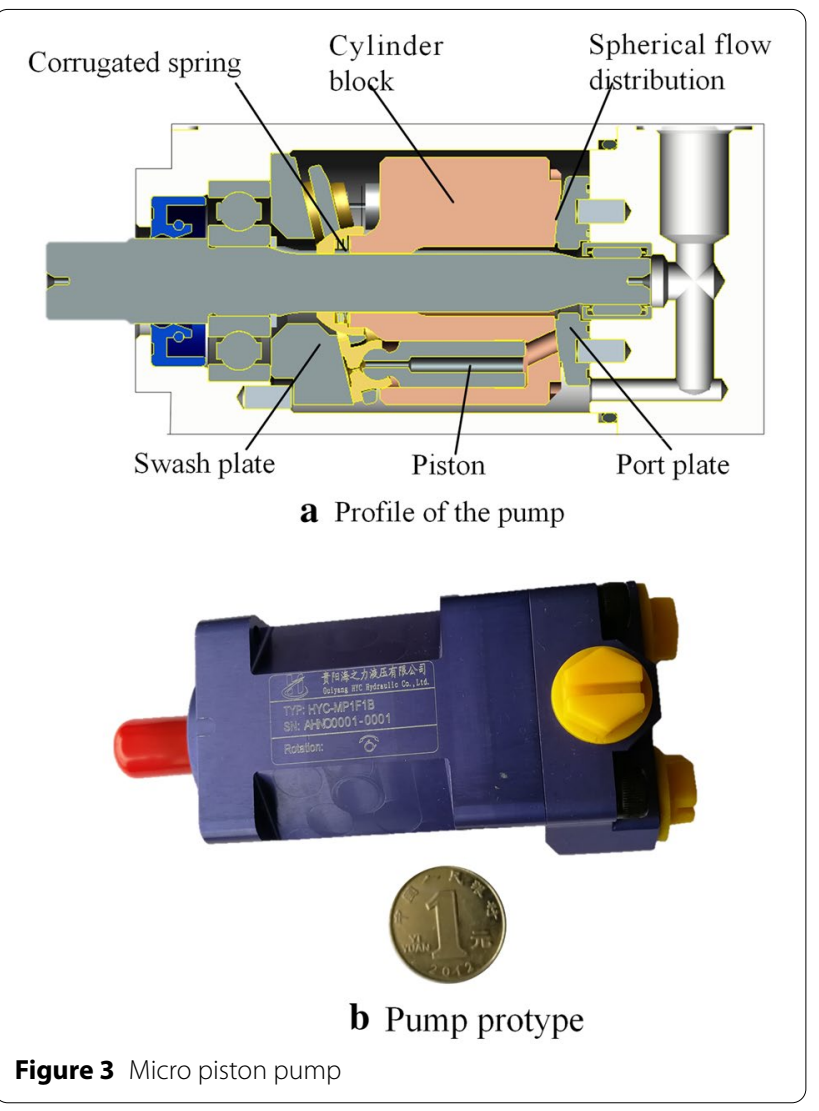

pushed together. The tapered surface (a) is the sealing contact with the valve housing (b). Oil flows from inlet port to outlet port 1 of the compact valve, the cylinder in the assisted mode (shown in Figure 6(b)). (III) When the system pressure exceeds the required pressure, only the valve spool 2 will be pushed, and the pressure will be released through the flow channel of the valve spool 2 .

The compact valve combines the functions of switching flow passage and releasing pressure, which is highly integrated, and weights only about $28 \mathrm{~g}$. More detail about the valve block was described in Refs. [26, 27].

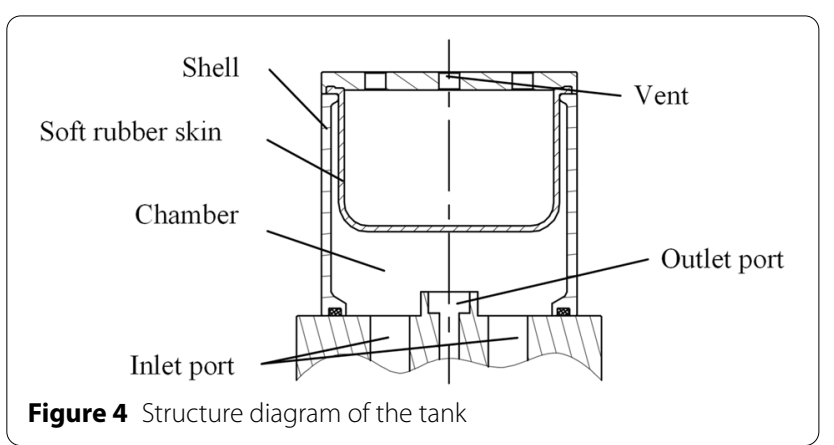




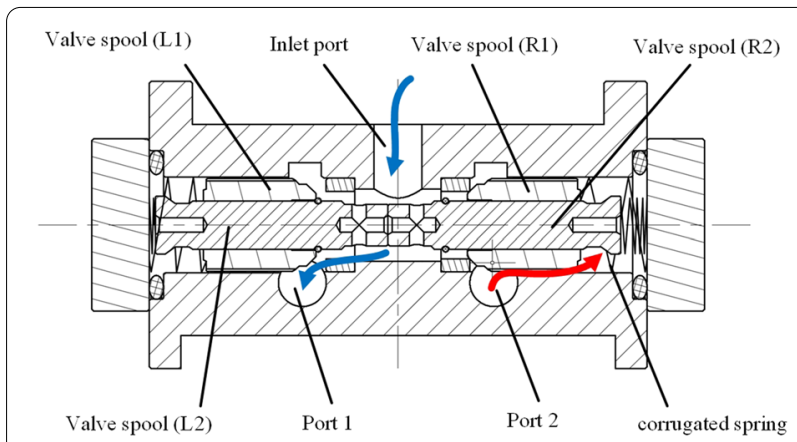

Figure 5 Structure diagram of FMV

\subsection{Key Parameters}

Considering the HAS is used for the joint actuation, its parameters should match the exoskeleton's performance requirements.

Figure 7(a) gives the structure of the hip joint, and Figure 7(b) shows the mechanism sketch of it. Where $O$ is the center of rotation of the hip joint, $a b$ is the length of the cylinder, $a c, b d, o c$, and $o d$ are the length of the hip joint linkage, $r_{i}$ is the distance from ends of the cylinder to $O$, and $\theta_{i}$ is the angle between the linkage and $r_{i}$. The cylinder length $a b$ can be obtained as follows:

$$
a b=\sqrt{r_{1}^{2}+r_{2}^{2}+2 r_{1} r_{2}\left(\theta+\theta_{1}+\theta_{2}\right)},
$$

where $\quad r_{1}=\sqrt{a c^{2}+c o^{2}}$, $\theta_{1}=\arctan \frac{a c}{c o}, \theta_{2}=\arctan \frac{b d}{o d}$.

The angular range of the hip joint comes from the data of Clinical Gait Analysis (CGA) [28]. The length ranges of the cylinder can be obtained by substituting the

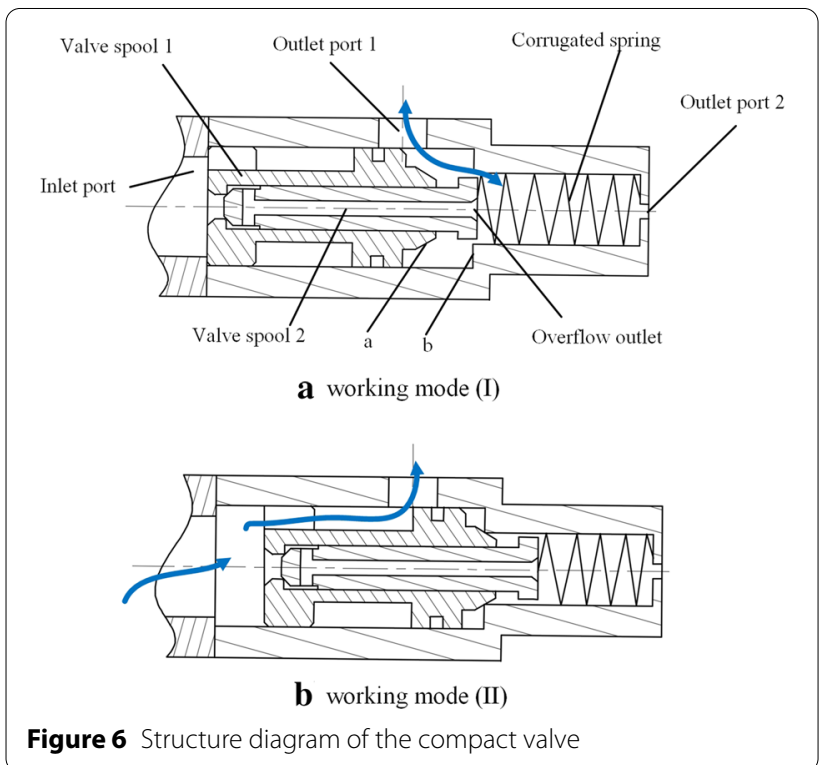

angular range into Eq. (1). It can be seen in the Figure 8, the hydraulic cylinder requires a maximum stroke of 58 $\mathrm{mm}$ in the walking cycle and a minimum stroke of $4 \mathrm{~mm}$ in the squatting.

Furthermore, by differentiating the line displacement curve, the maximum velocity of the cylinder can be obtained as $140 \mathrm{~mm} / \mathrm{s}$ (no load swing), which is an important parameter for the integrated ESM and pump. According to the simulation results, the maximum rotation speed of the ESM is determined as $4000 \mathrm{r} / \mathrm{min}$.

Through virtual simulation of the exoskeleton walking and squatting, the maximum force requirement for the hip joint is estimated, and the required pressure for the hydraulic system is also determined as $18 \mathrm{MPa}$. The walking and squatting simulation are carried out under the $50 \mathrm{~kg}$ load, and the simulation thrust data are shown in Figure 9. Due to the walking mode requires one leg to support the whole-body weight, and to resist the external impact, the hydraulic cylinder reaches to the maximum thrust of $1770 \mathrm{~N}$. The selected normal torque of the ESM is determined as $0.8 \mathrm{~N} \cdot \mathrm{m}$ and the peak torque is obtained about $1.8 \mathrm{~N} \cdot \mathrm{m}$. The HAS parameters of the exoskeleton hip joints are summarized in Table 1.

\section{Modeling of the HAS}

During the exoskeleton working, the rodless chamber of the cylinder (shown in Figure 2) always remains high pressure, and the position control of HAS is actually the flow control of the rodless (high pressure) chamber.

\subsection{Flow Functions}

Because the safety valve, DCV and FMV have sufficiently fast response, their dynamics are reasonably ignored in the modeling process. In addition, it is assumed that the

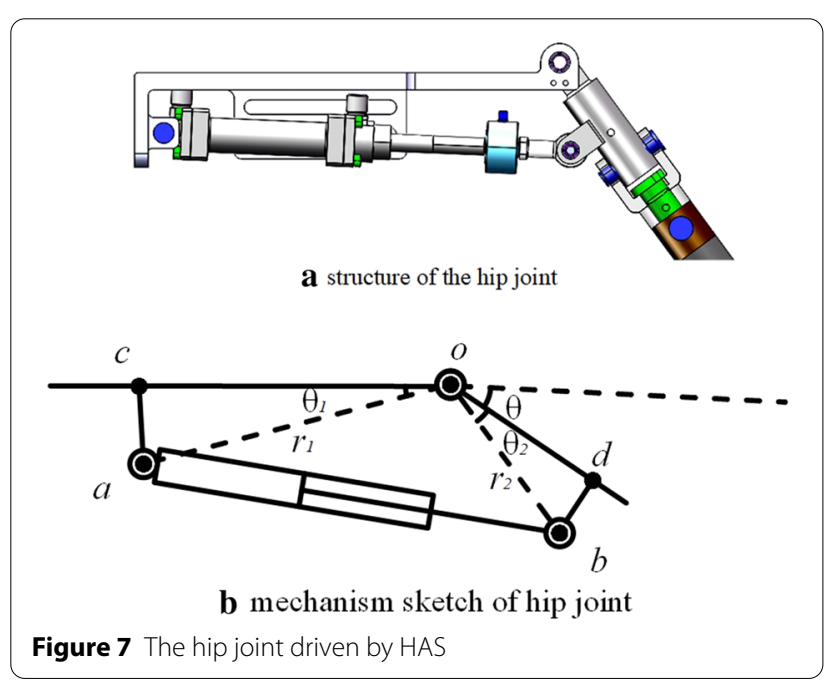




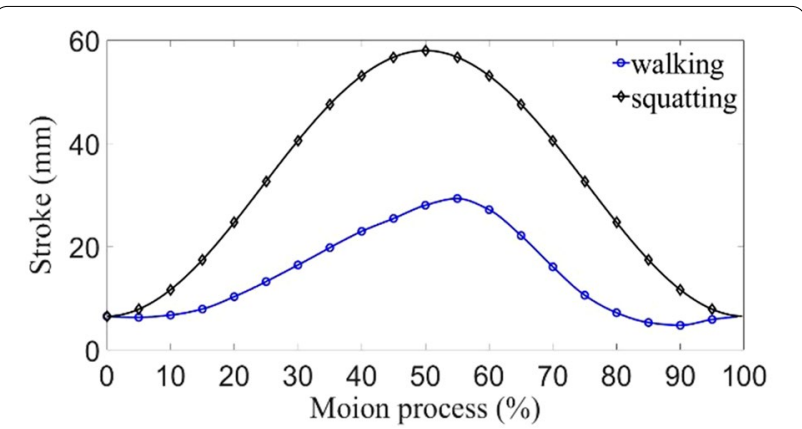

Figure 8 Stroke range in walking and squatting cycles

connection between the pump and the ESM is ideal. When the pressure is established $\left(p_{o}>p_{i}\right)$, the flow equations of the bidirectional piston pump are described as follows:

$$
\begin{aligned}
& Q_{o}=D_{p} n_{p}-L_{n}\left(p_{o}-p_{i}\right)-\frac{V_{A}+A_{A} x}{E y} \frac{\mathrm{d} p_{o}}{\mathrm{~d} t}, \\
& Q_{i}=D_{p} n_{p}-L_{n}\left(p_{o}-p_{i}\right)+\frac{V_{B}-A_{B} x}{E y} \frac{\mathrm{d} p_{i}}{\mathrm{~d} t},
\end{aligned}
$$

where $Q_{i}, Q_{o}$ are the inlet and outlet flow rates of the pump respectively; $D_{p}$ is the pump displacement; $n_{p}$ is the pump rotation speed; $C_{n}$ is the pump inner leakage coefficient; $p_{o}, p_{i}$ are the pump ports pressure connected to cylinder rodless and rod chambers, respectively; $A_{A}, A_{B}$ are the piston cross-sectional areas of the rodless and rod chambers respectively; $E_{y}$ is the oil elastic modulus, and $x$ is the displacement of the cylinder.

The flow rate equations for the rodless and rod chambers of the cylinder are described as follows:

$$
Q_{A}=A_{A} \frac{\mathrm{d} x}{\mathrm{~d} t}+\frac{V_{A}+A_{A} x}{E y} \frac{\mathrm{d} p_{A}}{\mathrm{~d} t}+L_{c}\left(p_{A}-p_{B}\right),
$$

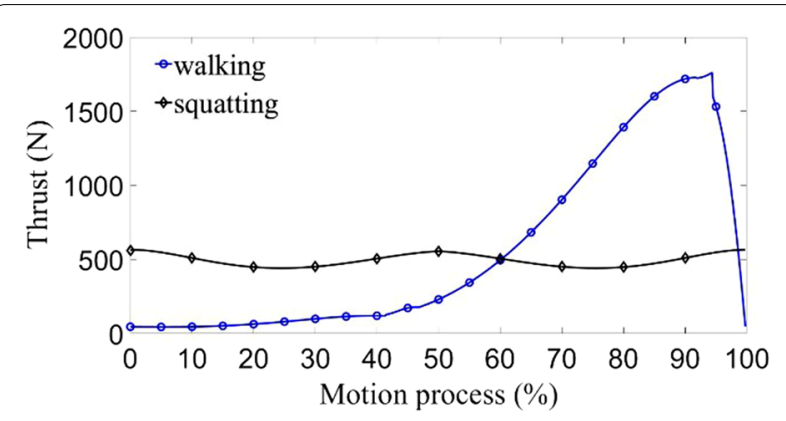

Figure 9 Thrust range in walking and squatting
Table 1 Specifications of the hip HAS

\begin{tabular}{ll}
\hline Parameters & Data \\
\hline Volumetric size & $52 \mathrm{~mm} \times 52 \mathrm{~mm} \times 245 \mathrm{~mm}$ \\
Weight & $2.5 \mathrm{~kg}$ \\
Stroke & $68 \mathrm{~mm}$ \\
Cylinder velocity & $160 \mathrm{~mm} / \mathrm{s}$ \\
Max. flow & $1.6 \mathrm{~L} / \mathrm{min}$ \\
Operating pressure & $18 \mathrm{MPa}$ \\
Inner diameter of cylinder & $14 \mathrm{~mm}$ \\
Rod diameter of cylinder & $10 \mathrm{~mm}$ \\
DC motor power & $340 \mathrm{~W}$
\end{tabular}

$$
Q_{B}=A_{B} \frac{\mathrm{d} x}{\mathrm{~d} t}-\frac{V_{B}-A_{B} x}{E y} \frac{\mathrm{d} p_{B}}{\mathrm{~d} t}-L_{c}\left(p_{A}-p_{B}\right),
$$

where $Q_{A}, Q_{B}$ are the flow of the rodless and rod chamber, $L_{c}$ is the cylinder inner leakage coefficient, and $p_{A}, p_{B}$ are the pressure of rodless and rod chambers, respectively.

The hose length between the pump and the cylinder is very short, so the losses of the pressure and flow is ignored. The pressure and flow rate between the inlet and outlet of the bidirectional piston pump and the rodless and rod chambers of the cylinder are assumed to the following relationship:

$$
\begin{gathered}
Q_{A}=Q_{o}, Q_{B}=Q_{i}, \\
p_{o}=p_{A}, p_{i}=p_{B} .
\end{gathered}
$$

\subsection{Dynamic Model of the HAS}

According to Newton's law, the dynamic equilibrium equation of the cylinder is given as

$$
M_{a} \ddot{x}=A_{A} p_{A}-A_{B} p_{B}-M_{L} \ddot{x}-B \dot{x},
$$

where $M_{a}, M_{L}$ are the mass of the cylinder piston and the load respectively, and $B$ is the piston motion damping coefficient.

\subsection{Transfer Function}

Integrating Eqs. (1)-(4), the system differential equation yields are obtained as:

$$
\begin{aligned}
& 2 D_{p} n_{p}-2 L_{n}\left(p_{o}-p_{i}\right)-\frac{V_{A}+A_{A} x}{E_{y}} \frac{\mathrm{d} p_{o}}{\mathrm{~d} t} \\
& =\left(A_{A}+A_{B}\right) \frac{\mathrm{d} x}{\mathrm{~d} t}+\frac{V_{A}+A_{A} x}{E_{y}} \frac{\mathrm{d} p_{A}}{\mathrm{~d} t} .
\end{aligned}
$$

The soft rubber skin isolates the oil from outside air, while the soft rubber skin directly connects with the 
atmosphere (shown in Figure 4). Therefore, the inlet pressure of the pump equals to the atmospheric pressure, i.e., $p_{i}=0$, so Eq. (9) can be written as:

$$
2 D_{p} n_{p}-2 L_{n} p_{o}-2 \frac{V_{A}+A_{A} x}{E_{y}} \dot{p}_{o}=\left(A_{A}+A_{B}\right) \dot{x} .
$$

When the piston of the cylinder moves near its center stoke position, the differential equation between the ESM rotation speed and the cylinder output displacement can be obtained by utilizing Eqs. (8) and (10):

$$
D_{p} n_{p}=\frac{V_{A} M}{E_{y} A_{A}} \dddot{x}+\left(\frac{L_{n} M}{A_{A}}+\frac{V_{A} B}{E_{y} A_{B}}\right) \ddot{x}+\left(\frac{L_{n} B}{A_{A}}+\frac{A_{A}+A_{B}}{2}\right) \dot{x} .
$$

The system transfer function between the HAS displacement and the ESM rotation speed is obtained from the differential Eq. (11). The open loop model of the HAS is shown in Figure 10 in the individual component models.

$$
\frac{x}{n_{p}}=\frac{\frac{A_{A} E_{y} D_{p}}{V_{A} M}}{s^{3}+\left(\frac{E_{y} L_{n}}{V_{A}}+\frac{B}{M}\right) s^{2}+\left(\frac{L_{n} E_{y} B}{V_{A} M}+\frac{A_{A} E_{y}\left(A_{A}+A_{B}\right)}{2 V_{A} M}\right) s} .
$$

From Eq. (12), the relationship between the HAS displacement $x$ and the ESM rotation speed $n_{p}$ is a thirdorder system. Furthermore, the relationship between the HAS velocity $\dot{x}$ and the $n_{p}$ can be known as a secondorder system. According to the characteristics of the second-order system, the natural frequency $\left(\omega_{n}\right)$ and speed amplification coefficient $(K)$ of the system can be analyzed. The $\omega_{n}$ of the HAS is obtained as

$$
\omega_{n}=\sqrt{\left(L_{n} B+\frac{\left(A_{A}+A_{B}\right) A_{A}}{2}\right) \frac{E_{y}}{V_{A} M}} .
$$

It can be seen that the natural frequency arises by increasing the oil elastic modulus $\left(E_{y}\right)$, piston areas $A_{A}$ and $A_{B}$, drops by increasing the volume $\left(V_{A}\right)$ and mass $(M)$.

The $K$ of the open loop transfer function is

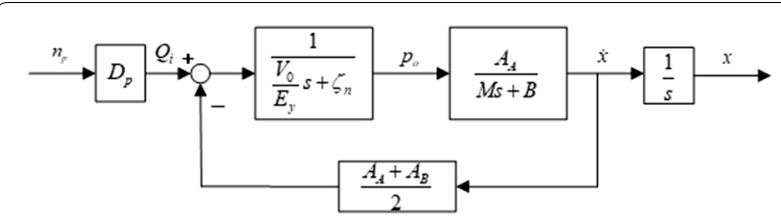

Figure 10 Block diagram of the HAS

$$
K=\frac{2 D_{p} A_{A}}{2 L_{n} B+\left(A_{A}+A_{B}\right) A_{A}} .
$$

It can also be seen that the speed amplification coefficient $K$ will decrease due to the $L_{n}$ existing.

\section{Identification of the HAS}

\subsection{Prototype of the HAS}

The HAS prototype for the hip joint of the exoskeleton is shown in Figure 11, its weight is about $2.5 \mathrm{~kg}$, including the mass of all sensors and hoses. Table 2 shows comparison between the HAS and the compact hydraulic power unit (CHPU) $[9,10]$. The total four HASs weighs $10 \mathrm{~kg}$, and the CHPU weighs $16.6 \mathrm{~kg}$ without the 4 cylinders and force sensors. The weight of the current hydraulic actuating system for the exoskeleton is reduced by about $40 \%$ and its power density increased by almost 1.6 times compared with the CHPU.

\subsection{System Identification of the HAS}

There are some parameters, such as the leakage coefficient $\left(L_{n}\right)$, actual elastic modulus of oil $\left(E_{y}\right)$, and friction coefficient $(B)$, which are difficult to be obtained accurately. Therefore, the identification of the system model is necessary.

The HAS is powered by lithium batteries and a STM32 processor is provided for the HAS control and data acquisition. In the test, the processor (STM32f4) sends rotation speed command to the motor controller (COPLEY) at a frequency of $200 \mathrm{~Hz}$. The cylinder position is measured by the displacement sensor (SMWEI, $5 \mathrm{~V}, 200$ $\mathrm{Hz}$ ), and the position is used as feedback signal. The

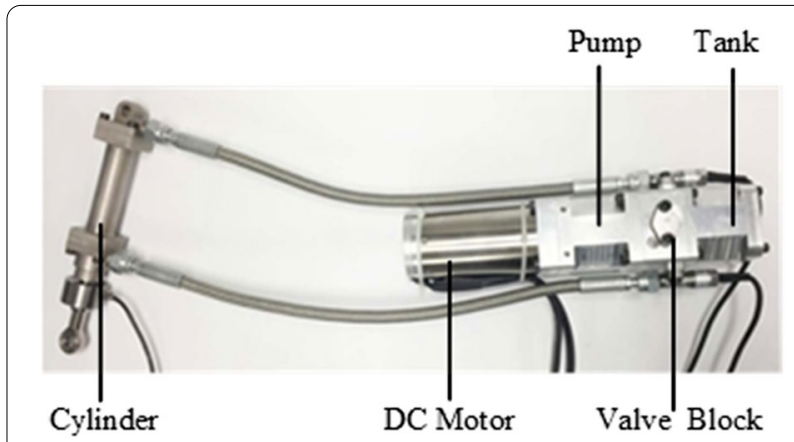

Figure 11 One HAS for the exoskeleton hip joint

Table 2 Comparison of hydraulic actuating systems

\begin{tabular}{lll}
\hline & CHPU & HASs \\
\hline Weight $(\mathrm{kg})$ & 16.6 & 10 \\
Volume $\left(\mathrm{mm}^{3}\right)$ & $450 \times 250 \times 290$ & $104 \times 104 \times 245$ \\
Power density $(\mathrm{kW} / \mathrm{kg})$ & 0.087 & 0.136 \\
\hline
\end{tabular}




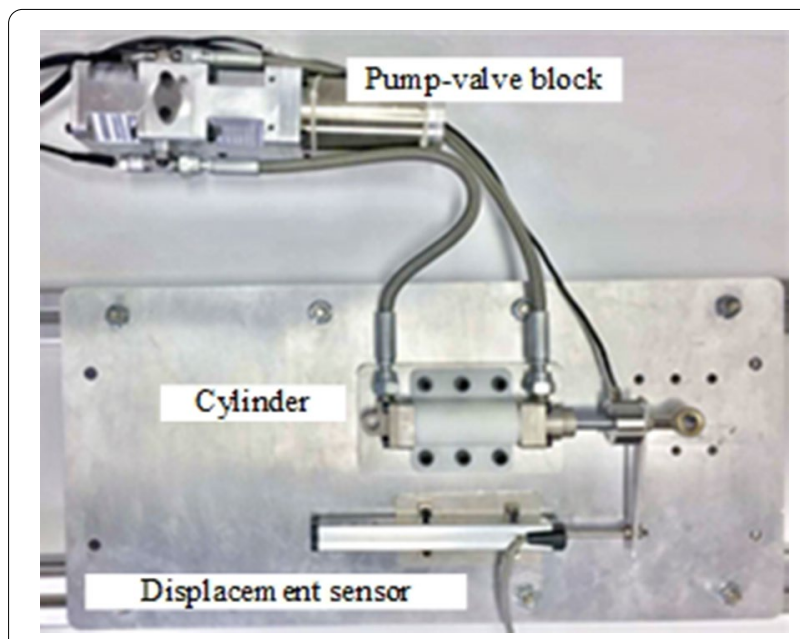

Figure 12 Test bench of HAS system identification

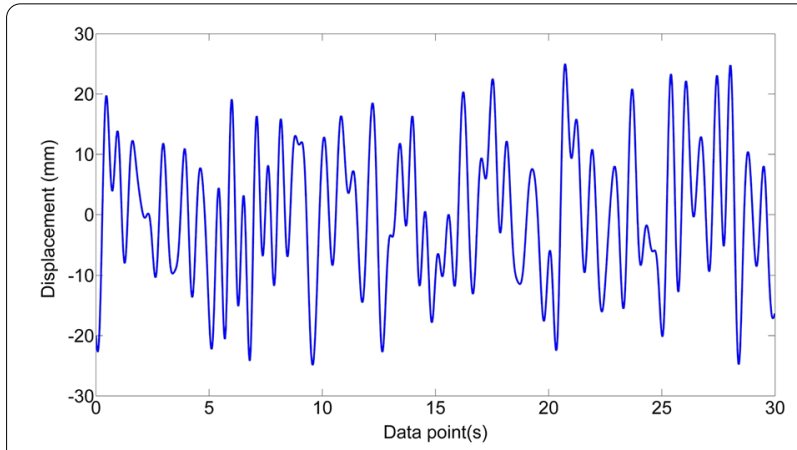

Figure 13 Sine input of the system identification

test bench of the HAS system identification is shown as Figure 12.

To achieve the reliable parameters of the system model, input-output data of the identification must capture the system dynamics. A sine curve with arbitrary frequency distribution range of $0-5 \mathrm{~Hz}$ and amplitude range of \pm 25 $\mathrm{mm}$ is selected as the input signal. Figure 13 shows the generated test 3000 data samples in $30 \mathrm{~s}$.

In the actual identification experiment, HAS performs tracking experiment according to the sine input under the closed-loop controller of $P=35$. In previous section analysis, the transfer function is a third-order system, which will be used as the prior identification model. The system closed-loop transfer function is identified by the least squares regression method [29]. According to datum of the sine input and the displacement output, the nominal open-loop transfer function is obtained by removing $P$ controller.

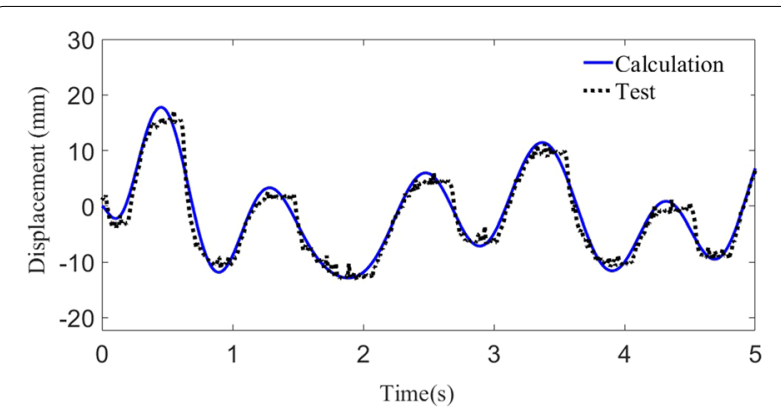

Figure 14 Model validation

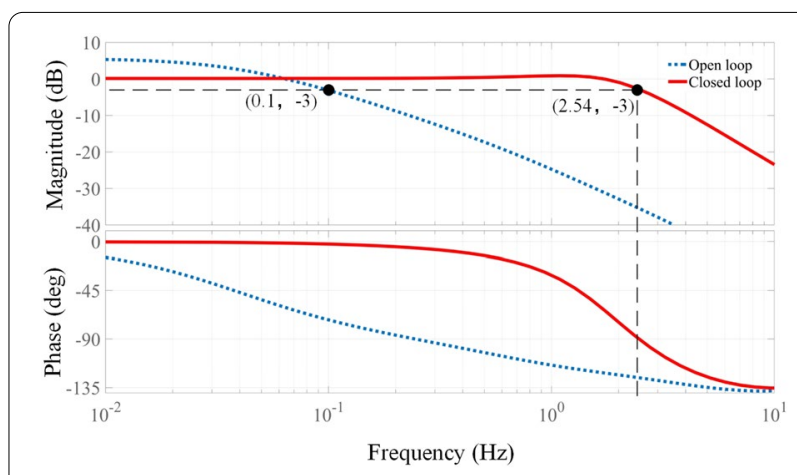

Figure 15 Frequency response

$$
G(s)=\frac{(s+149.38)(s+9.58)}{(s+0.24)(s+5.53)(s+26.21)} .
$$

Eq. (15) is the nominal open-loop transfer function of the HAS which reflects the relationship between the HAS displacement $x$ and the ESM rotation speed $n_{p}$. It can be judged that the HAS is a stable system according to all the poles distributed in the left half plane of the coordinate. However, the system is in a marginally stable state and susceptible to external disturbance.

A new set of sinusoidal superposition with the same frequency range and amplitude characteristics is generated to verify the nominal transfer function. As shown in Figure 14, the solid curve represents the calculation result, which is calculated from the Eq. (15) and the dot curve represents the test results. The fit degree of the model is $82.25 \%$. It shows that the output of the nominal transfer function (Eq. (15)) agrees with the test result well, which is sufficient for developing the linear control system. 


\section{Performance Tests of HAS}

\subsection{Frequency Response Test}

The system characteristic is further analyzed in the frequency response. Considering application, the test is carried out under $50 \mathrm{~kg}$ loading with sinusoidal inputs with $\pm 25 \mathrm{~mm}$ amplitude at different frequencies. Figure 15 shows the results of closed loop frequency response based on proportional differential $(P=35, D=15)$ controller. It shows that the closed loop system bandwidth of the HAS is about $2.54 \mathrm{~Hz}$ (solid curve). By comparing the open loop bode plot (dot curve) obtained from Eq. (15), PD control reconfigures the poles of the system, improves HAS stability, and ensures the system operating bandwidth.

\subsection{Trajectory Tracking}

Walking and squatting tasks are the two most common motion cycles for exoskeletons and these two motion trajectories are chosen to demonstrate whether the HAS can meet basic requirements of exoskeleton. The closedloop trajectory tracking tests are carried out under a load of $25 \mathrm{~kg}$. The stride frequency is $0.85 \mathrm{~Hz}$ and the squatting frequency is $0.5 \mathrm{~Hz}$, which are close to the normal adult motion data $[10,28,30]$.

The PD controller is tuned for the HAS position control. Figure 16 shows trajectory tracking test results. It is worth noting the resemblance between the generated signals are both in reference and test, which demonstrate the HAS has ability to be applied to the real exoskeleton.

\subsection{HAS Performance in the Exoskeleton}

In order to verify the actual performance of the HAS in the exoskeleton, the test with a $25 \mathrm{~kg}$ load was performed on an exoskeleton (in Figure 17). The test results are

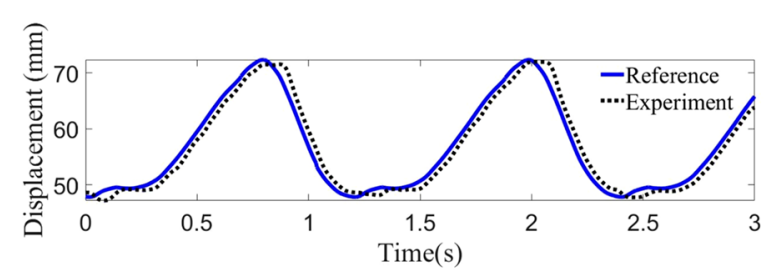

a walking trajectory tracking

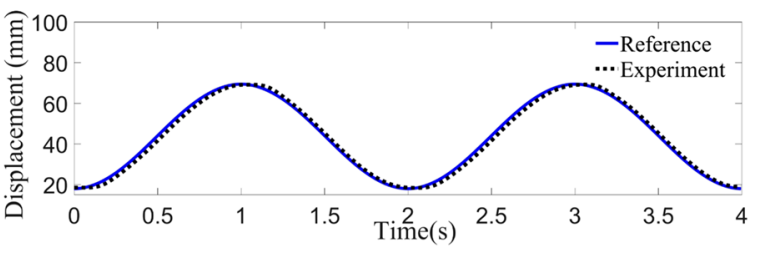

b squatting trajectory tracking

Figure 16 Walking and squatting tracking
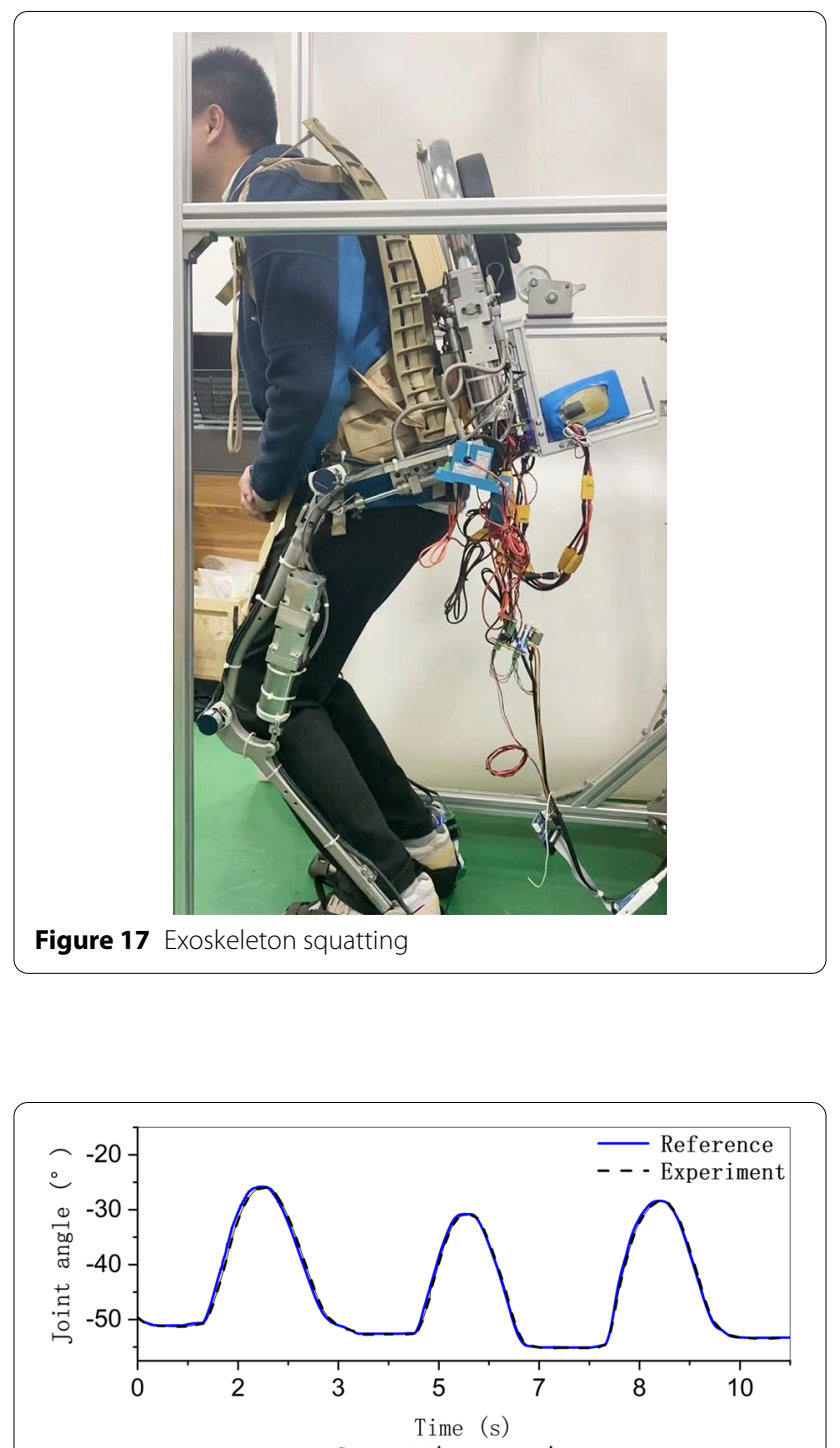

a squatting experiment

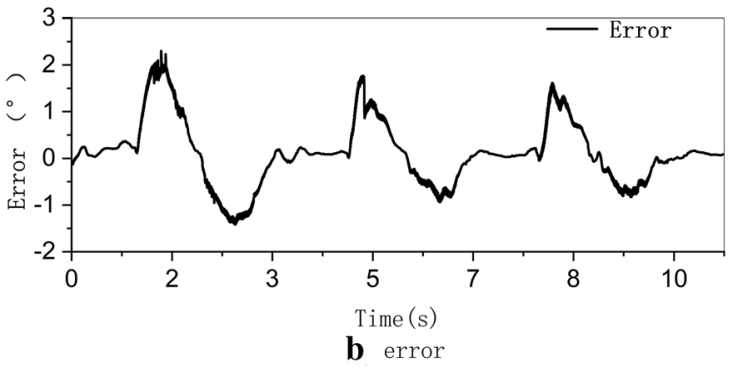

Figure 18 Squatting experiment results

shown in Figure 18. The target trajectory is determined by the human-exoskeleton interaction algorithm which is not described in detail here, and the position tracking is based on the PD control algorithm as before. The test 
results show that the hip joint driven by the HAS can follow the desired joint angle with $-1.5^{\circ}+2.5^{\circ}$ error during the robot squatting process.

\section{Conclusions}

(1) The innovative hydraulic actuating systems for the exoskeleton are developed successfully, the four HASs for the robot weighs only $10 \mathrm{~kg}$ including the mass of all the cylinders and sensors. The weight and power density of the hydraulic actuating system for the exoskeleton has been improved significantly.

(2) The dynamic characteristics of the HAS is analyzed, and the system identification shows the HAS has the good response frequency.

(3) The trajectory tracking tests in the exoskeleton demonstrate that novel HAS can meet motion requirements of the robot well.

\section{Acknowledgements}

Not applicable.

\section{Authors' Contributions \\ XO was in charge of the whole trial; MS wrote the manuscript; JM and HY assisted with discussion and modification during manuscript preparation. $\mathrm{GH}$ assisted in components design and manufacture. All authors read and approved the final manuscript.}

\section{Authors' Information}

Maowen Sun, born in 1991, is currently a PhD candidate at State Key Laboratory of Fluid Power \& Mechatronic Systems, Zhejiang University, China. He received his bachelor degree from Nanchang University, China, in 2017. His research interests include exoskeleton robot and control of electro-hydraulic actuator.

Xiaoping Ouyang, born in 1974, is currently a professor and a PhD candidate supervisor at State Key Laboratory of Fluid Power \& Mechatronic Systems, Zhejiang University, China. His main research interests include mechatronics engineering, fluid power transmission and control, exoskeleton robot.

Jouni Mattila, born in 1970, is currently a professor and a PhD candidate supervisor at Department of Intelligent Hydraulics and Automation, Tampere University, Tampere, Finland. His main research interests include hydraulics robotics, non-linear control.

Huayong Yang, born in 1961, is currently a professor and a PhD candidate supervisor at State Key Laboratory of Fluid Power \& Mechatronic Systems, Zhejiang University, China. His main research interests include mechatronics engineering, fluid power transmission and control.

Gang Hou, born in 1974, is currently head of Guiyang HYC Hydraulic Co., Ltd. specializing in the development of hydraulic components and hydraulic systems.

\section{Funding}

Supported by National Key R\&D Program of China (Grant No. 2018YFB1305400, 2018YFB1305402), National Natural Science Foundation of China (Grant No. 518902883), and Fundamental Research Funds for the Central Universities (Grant No. 2018XZZX001-04).

\section{Competing Interests}

The authors declare no competing financial interests.

\section{Author Details}

${ }^{1}$ State Key Laboratory of Fluid Power \& Mechatronic Systems, Zhejiang University, Hangzhou 310027, China. ${ }^{2}$ Department of Intelligent Hydraulics and Automation, Tampere University, 33101 Tampere, Finland. ${ }^{3}$ Guiyang HYC Hydraulic Co., Ltd, Guiyang 550018, China.

Received: 14 March 2020 Revised: 26 December 2020 Accepted: 11 January 2021

Published online: 11 March 2021

\section{References}

[1] A J Young, D P Ferris. State of the art and future directions for lower limb robotic exoskeletons. IEEE Transactions on Neural Systems and Rehabilitation Engineering, 2017, 25(2): 171-182.

[2] E Rocon, L José Pons. Introduction: Exoskeletons in Rehabilitation Robotics. Berlin, Heidelberg: Springer, 2011.

[3] J Zhang, P Fiers, K A Witte, et al. Human-in-the-loop optimization of exoskeleton assistance during walking. Science, 2017, 356(6344): 1280-1284.

[4] T Zhang, M Tran, H Huang. Design and experimental verification of hip exoskeleton with balance capacities for walking assistance. IEEE/ASME Transactions on Mechatronics, 2018, 23(1): 274-285.

[5] Zhixin Wang. Raytheon Launched XOS2 second-generation exoskeleton device. Light Weapons, 2010, 24: 44.

[6] A B Zoss, H Kazerooni, A Chu. Biomechanical design of the Berkeley lower extremity exoskeleton (BLEEX). Mechatronic IEEE/ASME Trans., 2006, 11:128-38.

[7] A Chu, H Kazerooni, A Zoss. On the biomimetic design of the Berkeley lower extremity exoskeleton (BLEEX). Proceedings of the 2005 IEEE International Conference on Robotics and Automation, IEEE, 2005: 4345-4352.

[8] R Angold, A B Zoss, JW Burns, et al. Hip and knee actuation systems for lower limb orthotic devices: U.S. Patent 9,011,354. 2015-4-21 [2020-12-10]. https://patents.google.com/patent/US9011354B2/en.

[9] X Ouyang, S Ding, B Fan, et al. Development of a novel compact hydraulic power unit for the exoskeleton robot. Mechatronics, 2016, 38: 68-75.

[10] B Q Fan. Research on the key technologies of the hydraulic lower limb exoskeleton robot. Hangzhou: Zhejiang University, 2017. (in Chinese)

[11] N Alle, S S Hiremath, S Makaram, et al. Review on electro hydrostatic actuator for flight control. International Journal of Fluid Power, 2016, 17(2): $1-21$.

[12] S Habibi, A Goldenberg. Design of a new high performance electrohydraulic actuator. 1999 IEEE/ASME International Conference on Advanced Intelligent Mechatronics (Cat. No. 99TH8399), IEEE, 1999: 227-232.

[13] K K Ahn, D N C Nam, M Jin. Adaptive backstepping control of an electrohydraulic actuator. IEEE/ASME Transactions on Mechatronics, 2013, 19(3): 987-995.

[14] Y Lin, Y Shi, R Burton. Modeling and robust discrete-time sliding-mode control design for a fluid power electrohydraulic actuator (EHA) system. IEEE/ASME Transactions on Mechatronics, 2011, 18(1): 1-10.

[15] Y Li, Z Jiao, TYu, et al. Viscous loss analysis of the flooded electro-hydrostatic actuator motor under laminar and turbulent flow states. Processes, 2020, 8(8): 975

[16] J P Henderson, A Plummer, N Johnston. An electro-hydrostatic actuator for hybrid active-passive vibration isolation. International Journal of Hydromechatronics, 2018, 1(1): 47-71.

[17] T Ko, H Kaminaga, Y Nakamura. Underactuated four-fingered hand with five electro hydrostatic actuators in cluster. 2017 IEEE International Conference on Robotics and Automation (ICRA), IEEE, 2017: 620-625.

[18] TKang, H Kaminaga, Y Nakamura. A robot hand driven by hydraulic cluster actuators. 2014 IEEE-RAS International Conference on Humanoid Robots, IEEE, 2014: 39-44.

[19] T Ko, H Kaminaga, Y Nakamura. Key design parameters of a few types of electro-hydrostatic actuators for humanoid robots. Advanced Robotics, 2018, 32(23): 1241-1252.

[20] H Kaminaga, T Ko, R Masumura, et al. Mechanism and control of whole-body electro-hydrostatic actuator driven humanoid robot hydra. International Symposium on Experimental Robotics, Springer, Cham, 2016 656-665. 
[21] Y K Lee, S J Lee. A bio-mimetic robot arm actuated by micro EHA. 2013 10th International Conference on Ubiquitous Robots and Ambient Intelligence (URAI), IEEE, 2013: 39-44.

[22] B Song, D Lee, S Y Park, et al. Design and performance of nonlinear control for an electro-hydraulic actuator considering a wearable robot. Processes, 2019, 7(6): 389.

[23] D Lee, B Song, S Y Park, et al. Development and control of an electrohydraulic actuator system for an exoskeleton robot. Applied Sciences, 2019, 9(20): 4295

[24] Xiaoping Ouyang, Maowen Sun, Zhenfei Li, et al. Distributed hydraulic power source lower limb exoskeleton robot. CN: ZL201811363810.8. 2020-05-22[2020-12-10]

[25] H Gang, Y Mao, M C Yuan, et al. Port plate of swash plate type plunger pump and motor and matching cylinder body. CN: ZL201510487098.2. 2018-0831[2020-12-10]. http://cpquery.sipo.gov.cn/txnPantentInfoList.do?inner -flag:open-type $=$ window\&inner-flag:flowno $=1607586751022$
[26] X Ouyang, H Gang, M C Yuan, et al. Power-assisted mode automatic switch-over valve and exoskeleton robot hydraulic transmission system. CN: 201810739480.1. 2018-07-06[2020-12-10]. http://cpquery.sipo.gov.cn/ txnPantentInfoList.do?inner-flag:open-type=window\&inner-flag:flown $\mathrm{O}=1607586751022$.

[27] H Gang, HW Bo, C Xiong, et al. Flow matching valve suitable for asymmetric closed loop. CN: 201810421229.0. 2018-05-04. http://cpquery.sipo.gov.cn/ txnPantent|nfoList.do?inner-flag:open-type=window\&inner-flag:flown $\mathrm{O}=1607586751022$.

[28] CGA Normative Gait Database [OL]. http://ww.clinicalgaitanalysis.com/ data/index.html.

[29] Z Pang. System identification and adaptive control MATLAB simulation. Beijing: Beihang University Press, 2009. (in Chinese)

[30] D A Winter. Biomechanics and motor control of human movement. Waterloo: University of Waterloo Press, 1987

\section{Submit your manuscript to a SpringerOpen ${ }^{\circ}$ journal and benefit from:}

- Convenient online submission

- Rigorous peer review

- Open access: articles freely available online

- High visibility within the field

- Retaining the copyright to your article

Submit your next manuscript at springeropen.com 